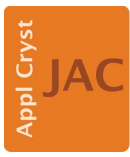

JOURNAL OF APPLIED CRYSTALLOGRAPHY

ISSN 1600-5767

Received 12 April 2019

Accepted 20 May 2019

Edited by D. I. Svergun, European Molecular Biology Laboratory, Hamburg, Germany

Keywords: nanoporous alumina; small-angle neutron scattering; nanochannels; structural characterization.

Supporting information: this article has supporting information at journals.iucr.org/j

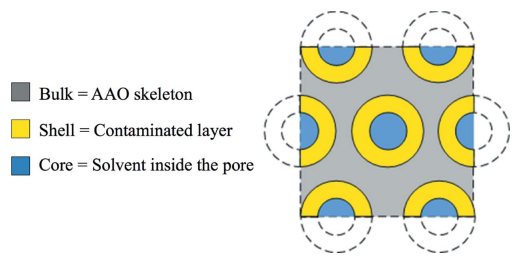

OPEN $\odot$ ACCESS

\section{A novel methodology to study nanoporous alumina by small-angle neutron scattering}

\author{
Anastasia Christoulaki, ${ }^{a}$ Alexis Chennevière, ${ }^{b}$ Isabelle Grillo, ${ }^{c}$ Lionel Porcar, \\ Emmanuelle Dubois ${ }^{\mathrm{a}}$ and Nicolas Jouault ${ }^{\mathrm{a} *}$
}

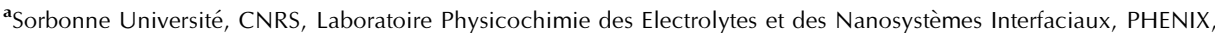
F-75005 Paris, France, 'baboratoire Léon Brillouin (LLB), CEA Saclay, 91191 Gif-Sur-Yvette, France, and ${ }^{\mathbf{c}}$ Institut LaueLangevin (ILL), 71 avenue des Martyrs, CS 20156, 38042 GRENOBLE Cedex 9, France. *Correspondence e-mail: nicolas.jouault@sorbonne-universite.fr
\end{abstract}

Nanoporous anodic aluminium oxide (AAO) membranes are promising host systems for confinement of condensed matter. Characterizing their structure and composition is thus of primary importance for studying the behavior of confined objects. Here a novel methodology to extract quantitative information on the structure and composition of well defined AAO membranes by combining small-angle neutron scattering (SANS) measurements and scanning electron microscopy (SEM) imaging is reported. In particular, (i) information about the pore hexagonal arrangement is extracted from SEM analysis, (ii) the best SANS experimental conditions to perform reliable measurements are determined and (iii) a detailed fitting method is proposed, in which the probed length in the fitting model is a critical parameter related to the longitudinal pore ordering. Finally, to validate this strategy, it is applied to characterize AAOs prepared under different conditions and it is shown that the experimental SANS data can be fully reproduced by a core/shell model, indicating the existence of a contaminated shell. This original approach, based on a detailed and complete description of the SANS data, can be applied to a variety of confining media and will allow the further investigation of condensed matter under confinement.

\section{Introduction}

The confinement of condensed matter in nanoporous media can induce at the nanoscale drastic structural or dynamical changes that ultimately lead to original properties that are not achievable under bulk conditions (Huber, 2015). Such behavior can be then exploited to design new devices or materials with enhanced properties (Md Jani et al., 2013). Among various nanoporous materials that can be used as a confining medium (clays, mesoporous silica, Vycor glass, nanotubes ... ), nanoporous anodic aluminium oxide (AAO) membranes are very interesting host systems for confinement studies (Shin et al., 2007; Krutyeva et al., 2013). AAOs are obtained through the controlled anodization of aluminium in acidic electrolytes (Masuda \& Fukuda, 1995) and are composed of high-aspectratio parallel cylindrical channels with perfectly tunable pore diameters $\left(D_{\mathrm{p}}\right.$ from 10 to $\left.100 \mathrm{~nm}\right)$, interpore distances $\left(D_{\mathrm{int}}\right.$ from 20 to $200 \mathrm{~nm}$ ), lengths ( $L_{\mathrm{p}}$ up to $500 \mu \mathrm{m}$ ) and porosities. AAO nanoporous materials are frequently used thanks to their numerous applications in which the control of structural parameters and composition is important: biosensing (Xiao et al., 2016), templating for the growth of functional materials (Lee \& Park, 2014; Sousa et al., 2014), and filtration or chemical separation devices (Mozalev et al., 2001). 
A still challenging issue is to find experimental strategies to fully describe the confining medium. The pore-size distribution and porosity of porous materials can be investigated on scales from nanometres to hundreds of micrometres by different methods (Anovitz \& Cole, 2015). Most of the previous work done on AAOs (Le Coz et al., 2010; Napolskii et al., 2012; Lee \& Park, 2014) used direct imaging techniques [scanning or transmission electron microscopy (SEM or TEM) and atomic force microscopy] coupled with energy dispersive $\mathrm{X}$-ray (EDX) spectroscopy to characterize the AAOs. These techniques provide information about the pore surface organization and composition as well as section views of nanochannels. However, they have some limitations, such as the limited area studied and the impossibility of having access to the composition inside the whole sample.

Combining these direct imaging methods with scattering techniques can overcome these issues by giving access to averaged structural information over a larger volume (Gommes et al., 2016) and can also provide information about the pore orientation in both transverse and longitudinal directions. X-rays (via scattering or diffraction) have been mainly used to characterize the pore arrangement and longrange ordering (Dore et al., 2002; Engel et al., 2009; Napolskii et al., 2010; Waheed et al., 2011; Roslyakov et al., 2013, 2016) by tilting the sample along the vertical or horizontal axis normal to the beam. In particular, the different longitudinal pore orientations can be extracted (Roslyakov et al., 2013), and a strong correlation between transverse and longitudinal pore ordering has been observed (Roslyakov et al., 2016).

However, the internal composition is still not available. To that end, small-angle neutron scattering (SANS) appears to be a powerful tool to characterize both the pore organization and the chemical composition by determining the different scattering length densities (SLDs) of the material using the contrast variation method (Marchal \& Demé, 2003; Lagrené \& Zanotti, 2008; Grigoriev et al., 2010; Lefort et al., 2011). This method consists of canceling the confining medium scattering using an appropriate mixture of deuterated/hydrogenated solvents whose SLD matches that of the confining medium, known as the 'matching point'. Such an approach allows the determination of the composition of the material. However previous work done on AAO membranes showed that AAOs cannot be perfectly matched (Marchal \& Demé, 2003; Lagrené $\&$ Zanotti, 2008). The contrast variation shows a minimum but the scattering intensities remain high. Imperfect matching comes from inhomogeneities in composition in the AAO due to the presence of contaminants (anions from the electrolyte or water molecules) that organize as a shell around the pores (Mata-Zamora \& Saniger, 2005; Le Coz et al., 2010; Han et al., 2013). Perfect matching is thus not experimentally achievable and the scattering signal becomes more difficult to analyze. So far, owing to such complexities, few studies have attempted to fully reproduce the experimental scattering data. Marchal et al. (2001) fitted the SANS high- $q$ range of commercial $60 \mu \mathrm{m}$ thick anodic membranes by a Porod law to extract their specific surface. Lefort et al. (2011) used a combination of a cylinder form factor with a hexagonal structure factor to fit a homemade AAO under one contrast condition, considering that AAOs are homogeneous in composition. The only attempt to fit the data with a core/shell model was made by Engel et al. (2009) on AAOs measured in air by SAXS, but they did not discuss the obtained SLD values.

Here one has to mention the multiple scattering (MS) effects. MS is the probability for a neutron to be scattered several times and specifically arises in nanoporous materials because of the great length and number of nanopores (Marchal \& Demé, 2003; Grigoriev et al., 2010; Turkevych et al., 2012). MS creates an additional $q$-dependent contribution to the total scattering which progressively becomes dominant compared with the AAO scattering. Although some data correction strategies have been proposed in a few studies dealing with weak MS (Goyal et al., 1983), MS is generally undesirable because this high extra contribution cannot be treated in the context of the single scattering approximation and analytically corrected (Grillo, 2008). As a consequence the fitting of the scattering curve is mostly impossible.

In this context, this paper aims to describe a detailed strategy to extract quantitative information about the AAO structure and composition. We determine the best experimental conditions to avoid MS and discuss a fitting method, combining SEM analysis and SANS measurements, that leads to a detailed and complete description of the SANS data. This original approach, which can be applied to a variety of confining media, is a required step to further investigate the behavior of condensed matter under confinement.

\section{Experimental methods}

\subsection{AAO synthesis}

AAO was prepared using the classical two-step anodization (Masuda \& Fukuda, 1995) of pure aluminium. High-purity aluminium foil (Al, $0.32 \mathrm{~mm}$ thick, 99.999\% from Goodfellow) was rinsed with acetone and electropolished in a solution of ethanol/perchloric acid $(75: 25 \mathrm{v} / \mathrm{v})$ under $15 \mathrm{~V}$ for $30 \mathrm{~s}$. Then a first anodization was carried out over a period of $2 \mathrm{~h}$ in $0.3 \mathrm{M}$ oxalic acid (OA, anhydrous from Alfa Aesar) at different temperatures $\left(4,10,18\right.$ or $\left.22^{\circ} \mathrm{C}\right)$ under a constant voltage of $40 \mathrm{~V}$. After this first anodization, the formed oxide was removed in phosphochromic acid $\left(6 \mathrm{wt} \% \mathrm{H}_{3} \mathrm{PO}_{4}\right.$ and $1.8 \mathrm{wt} \%$ $\left.\mathrm{CrO}_{3}\right)$ at $60^{\circ} \mathrm{C}$ over a period of $2 \mathrm{~h} 30 \mathrm{~min}$, the $\mathrm{Al}$ substrate keeping the imprints of the nanopores. A second-step anodization was then performed under the same conditions, leading to the formation of self-ordered AAOs. Note that alumina grows normal to the $\mathrm{Al}$ foil, producing an equivalent $\mathrm{AAO}$ thickness on both sides of the foil. The total AAO thicknesses, which take into account both sides, were determined by SEM (see Table 1) and can be tuned by varying the anodization temperature and time. The temperature affects the current density $j$ and thus the AAO growth rate, and, for a given temperature, a longer anodization time produces thicker AAOs. Table 1 presents the different experimental conditions used in this study. We choose as sample name nomenclature OA-temperature (in ${ }^{\circ} \mathrm{C}$ )-time (in $\mathrm{h}$ ). 
Table 1

Experimental conditions used during the different anodizations.

The electrolyte was $0.3 \mathrm{M}$ oxalic acid (OA) and the voltage was fixed at $40 \mathrm{~V}$ for all our experiments.

\begin{tabular}{lllll}
\hline Names & Temperature $\left({ }^{\circ} \mathrm{C}\right)$ & Time $(\mathrm{h})$ & $j\left(\mathrm{~mA} \mathrm{~cm}^{-2}\right)$ & $L_{\mathrm{p}}(\mu \mathrm{m}) \dagger$ \\
\hline OA-4-4 & 4 & 4 & 1.90 & 23 \\
OA-10-4 & 10 & 4 & 2.96 & 36 \\
OA-18-4 & 18 & 4 & 4.60 & 58 \\
OA-22-4 & 22 & 4 & 6.90 & 84 \\
OA-18-11 & 18 & 11 h 25 min & 4.40 & 171 \\
\hline
\end{tabular}

\subsection{Scanning electron microscopy}

Information about the nanochannel length, pore size and organization was obtained by SEM imaging performed on a field emission gun scanning electron microscope (FEGSEM, SU-70 Hitachi). Observations were carried out in low-voltage and low-current conditions in order to characterize the insulating sample surface without any coating. Several accelerating voltages were tested, and the best image quality was obtained between acceleration voltages of 1 and $3 \mathrm{kV}$, while it was not possible to acquire images at lower and higher voltages because of high noise and charging effects, respectively. The pore diameters were determined using the $3 \mathrm{kV}$ images, because they provided deeper beam penetration and better brightness. Images with different magnifications $(\times 10000$, $\times 20000, \times 50000$ and $\times 100000$ ) were recorded to provide information about the long-range pore arrangement and a good pore-size resolution.

EDX spectroscopy measurements were also performed on the samples for elemental surface determination. The spectrometer used was an OXFORD X-Max SDD (crystal $50 \mathrm{~mm}^{2}$ ). The device was mounted on the FEGSEM. The software was an INCA version using XPP modeling for spectrum analysis. Analyses were performed at $5 \mathrm{kV}$ on uncoated specimens and no charging effect was observed. Standard references were used for quantification.

\subsection{Small-angle neutron scattering}

The pore organization and the AAO composition can be probed by SANS from the analysis of the scattering intensity and the determination of the AAO SLD. For aligned anisotropic objects with low size dispersity the scattering intensity $I(q)$ [with the magnitude of the scattering vector $q$ defined as $q=(4 \pi / \lambda) \sin \theta$, where $\theta$ is half the scattering angle and $\lambda$ is the wavelength of the incident neutrons] in the local monodisperse approximation (Pedersen, 1994) is generally expressed by the relation (1):

$$
I(q)=(\Phi / V)\left\langle F(q)^{2}\right\rangle S(q)
$$

with $\Phi$ the volume fraction of scattering objects (here nanopores), $V=\pi\left(D_{\mathrm{p}} / 2\right)^{2} L_{\mathrm{p}}$ the volume of the nanopore, $\langle F(q)\rangle$ the averaged scattering amplitude of the object form and $S(q)$ the structure factor. Our strategy to fit the $I(q)$ of AAOs will be detailed below.
SANS measurements were performed on the PAXY spectrometer at Laboratoire Léon Brillouin (CEA Saclay, France) and the D11 spectrometer at Institut Laue-Langevin (ILL, Grenoble, France). On PAXY, three configurations were used: $6.7 \mathrm{~m} / 15 \AA, 3 \mathrm{~m} / 6 \AA$ and $1 \mathrm{~m} / 6 \AA$, covering a $q$ range from $2 \times$ $10^{-3}$ to $0.5 \AA^{-1}$. Data reduction was performed with the inhouse PASINET software using the standard procedure (Brûlet et al., 2007), in which the sample thickness (which corresponds here to the pore channel length) and transmission (Tr) are required. On D11, two runs were performed (Jouault et al., 2016; Chennevière et al., 2018) and four configurations were used: $39 \mathrm{~m} / 6 \AA$ covering a $q$ range from $2 \times 10^{-3}$ to $0.4 \AA^{-1}$. Data reduction was performed using the ILL GRASP or Lamp software (Dewhurst, 2018; Richard et al., 1996).

Membranes of around $1 \times 1 \mathrm{~cm}$ with AAO present on both sides of the $\mathrm{Al}$ foil were measured either in air or in $\mathrm{H}_{2} \mathrm{O} / \mathrm{D}_{2} \mathrm{O}$ mixtures. For measurements in solvent, the AAOs were immersed in the mixtures for some seconds to be wetted and to avoid the presence of air bubbles, and then placed in circular cells between quartz windows separated by a $300 \mu \mathrm{m}$ Teflon o-ring corresponding to the total sample thickness. The cells were then aligned to have the pore channels oriented parallel to the neutron beam. The pore alignment was done by evaluating the isotropy of the $2 \mathrm{D}$ scattering pattern on the detector. Ideally, such patterns consist of concentric rings for a perfectly aligned sample. To precisely achieve this, the following procedure, depicted in Fig. S1 in the supporting information, was used. The sample was tilted in one direction ( $X$ or $Y$ ) to create strong anisotropy and the appearance of non-aligned spots in the other direction ( $Y$ or $X$ ). The alignment of the spots in this direction was then ensured by rotating the sample stepwise using a goniometer. This operation was repeated in the other direction, and the final alignment was inspected by performing a fast measurement to check the pattern isotropy. The spectra were then treated as isotropic using the whole detector.

Finally, since the AAO coherent scattering becomes negligible at high $q$, the incoherent background was subtracted by removing a constant value corresponding to the flat incoherent signal from the solvent, i.e. the constant value at high $q$. Note that measurement of AAO in air showed that the incoherent signal from the AAO is very low [around $10^{-3} \mathrm{~cm}^{-1}$, see Fig. 2(b)].

\section{Results and discussion}

In the following the detailed strategy to model the SANS data will be described step by step. This approach is based on the combination of SEM analysis to extract the structure factor $S(q)$ and SANS experiments performed in optimal conditions to extract quantitative parameters on the nanostructure and composition.

\subsection{Determination of the structure factor $S(q)$ by SEM analysis}

As shown by relation (1), $S(q)$ contributes to the SANS scattering intensity and can be independently determined by 
SEM image analysis. Fig. 1(a) shows a SEM image of the top surface of AAO membrane OA-18-4. The fully open nanopores are hexagonally ordered in domains of average lateral size $D$ with different orientations. From SEM analysis (i.e. image binarization followed by a pore-size distribution analysis), we find a pore diameter $\left(D_{\mathrm{p}}\right)$ of $42.0 \pm 3.8 \mathrm{~nm}$ (relative pore distribution $\sigma_{\mathrm{p}}=0.09$ ), a porosity $(P)$ of $14 \%$ and a pore density of $1.0 \times 10^{10}$ pores $\mathrm{cm}^{-2}$. Similar analyses were performed on all AAO samples (SEM images are shown in Fig. S2), and Table 2 summarizes all the different characteristic sizes. First, the anodization time affects the pore diameter and porosity as the sample is gradually dissolved with time by the acidic electrolyte (OA-18-4 versus

(a)

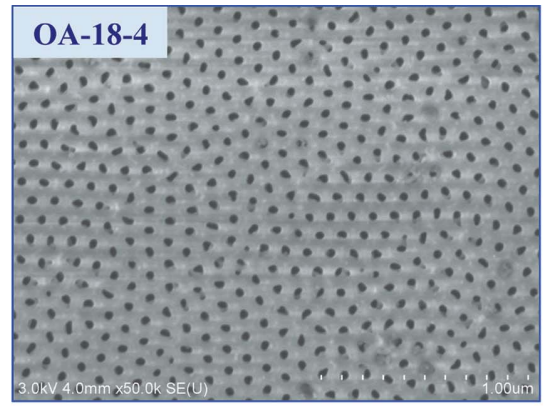

(b)

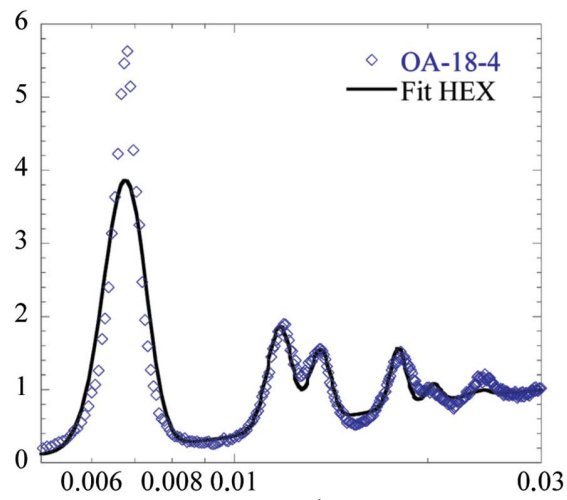

(c)

$\mathrm{q}\left(\AA^{-1}\right)$
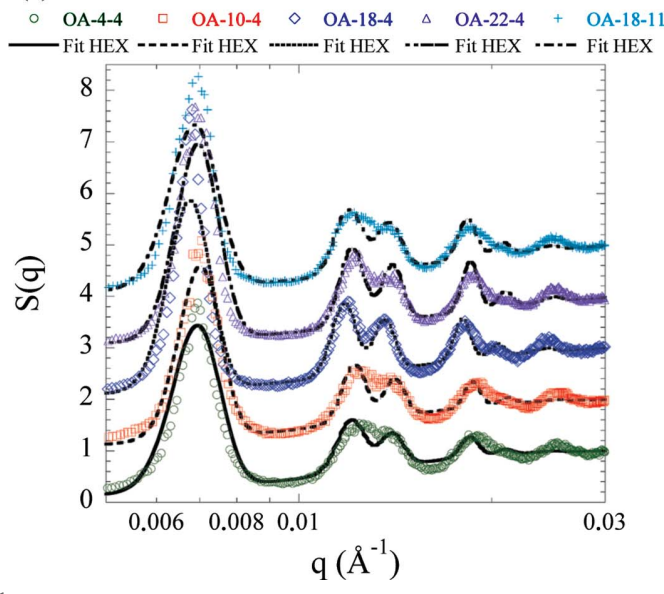

Figure 1

(a) SEM top surface image of OA-18-4. (b) Structure factor $S(q)$ derived from SEM-image FFT for OA-18-4 (blue circles). The continuous black line corresponds to the best fit using a hexagonal model. (c) $S(q)$ of all AAO samples. From bottom to top: OA-4-4, OA-10-4, OA-18-4, OA-22-4 and OA-18-11. The curves have been vertically shifted for clarity.
Table 2

Characteristic sizes of AAOs obtained by SEM analysis.

\begin{tabular}{llllll}
\hline Name & OA-4-4 & OA-10-4 & OA-18-4 & OA-22-4 & OA-18-11 \\
\hline$D_{\mathrm{p}}(\mathrm{nm})$ & 35 & 33 & 42 & 45 & 53 \\
$\sigma_{\mathrm{p}}$ & 0.16 & 0.08 & 0.09 & 0.05 & 0.14 \\
Pore density $\left(10^{10} \mathrm{~cm}^{-2}\right)$ & 1.1 & 1.1 & 1.0 & 1.0 & 1.0 \\
Porosity $(P)(\%)$ & 11 & 9 & 14 & 16 & 23 \\
$L_{\mathrm{p}}(\mu \mathrm{m})$ & 23 & 36 & 58 & 84 & 171 \\
$D_{\text {int }}(\mathrm{nm})$ & 103.4 & 102.6 & 106.4 & 103.4 & 104.5 \\
$\sigma_{\mathrm{a}}$ & 0.076 & 0.072 & 0.063 & 0.059 & 0.060 \\
$c_{\mathrm{L}}$ & 6.90 & 6.19 & 6.48 & 6.57 & 6.20 \\
$\delta\left(\times 10^{-4} \AA^{-1}\right)$ & 9.5 & 8.4 & 8.2 & 8.5 & 9.5 \\
Domain size $D(\mathrm{~nm})$ & 661 & 748 & 766 & 698 & 661 \\
\hline
\end{tabular}

OA-18-11). Then, for a given anodization time, $D_{\mathrm{p}}$ and $P$ increase with temperature while the pore density remains almost unchanged, as already observed in the literature (Lee \& Park, 2014).

To go further and quantify the pore arrangement and ordering in the transverse direction, the 2D fast Fourier transform (FFT) of the pore-center positions from SEM images at low magnification $(\times 10000)$ was performed to compute the structure factor $S(q)$ [see Fig. 1(b) for OA-18-4]. $S(q)$ presents different peaks whose positions are directly related to the pore ordering. Depending on the degree of pore ordering, $S(q)$ can be reproduced by either a hard-disk model for less ordered AAO (Rosenfeld, 1990; Engel et al., 2009) or a hexagonal model for clearly ordered AAO (Engel et al., 2009). Here $S(q)$ shows characteristic peaks of a hexagonal lattice and can be fitted assuming a hexagonal pore arrangement with a lattice parameter $a$ (i.e. the interpore distance $D_{\text {int }}$ ) expressed as follows (Förster et al., 2005):

$$
S(q)=1-G(q)+G(q) \frac{2 c_{\mathrm{L}}}{3^{1 / 2} a^{2} q} \sum m_{h k} L_{h k}(q) .
$$

$G(q)$ is the Debye-Waller factor, which is a disorder parameter taking into account the distortion of the lattice through the relative interpore distance distribution $\sigma_{a}$ :

$$
G(q)=\exp \left(-\sigma_{a}^{2} a^{2} q^{2}\right) .
$$

And $L_{h k}(q)$ is the normalized peak shape Gaussian function:

$$
L_{h k}(q)=\frac{2}{\delta \pi} \exp \left[-\frac{4\left(q-q_{h k}\right)^{2}}{\pi \delta^{2}}\right],
$$

with $\delta$ the peak width related to the domain size $D$ as $D=2 \pi / \delta$. In relation (2), $m_{h k}$ is the multiplicity factor (here fixed to 3 ) and $c_{\mathrm{L}}$ is a correction factor for the Porod invariant, which will be a free parameter in our fitting strategy (Sundblom et al., 2009).

Equation (2) fits the experimental $S(q)$ of OA-18-4 as seen by the continuous line in Fig. $1(b)$ with $D_{\text {int }}=106.4 \mathrm{~nm}, \sigma_{a}=$ 0.063 and $\delta=8.2 \times 10^{-4} \AA^{-1}$, giving a domain size $D$ of $766 \mathrm{~nm}$. Note that the amplitude of the first peak is not perfectly reproduced by the model. It can be better adjusted (curve not shown) by decreasing $\delta$ to $6 \times 10^{-4} \AA^{-1}$, i.e. $D=$ $1047 \mathrm{~nm}$, and increasing $\sigma_{a}$ to 0.072 . However, using these parameters, the adequacy of the model for the other peaks is lost. This can be explained by the fact that the experimental 
$S(q)$ contains information about the polydispersity of transverse pore positions and also about the polydispersity of the transverse orientations of ordered domains. Thus we chose to reproduce all the peaks to have a better picture of the pore ordering.

All the produced AAOs can be fitted with the hexagonal model as shown in Fig. 1(c). The different fitting parameters are listed in Table 2. The AAOs all adopt a hexagonal organization with similar interpore distance (which is fixed by the voltage during anodization) and domain size, in good agreement with previous work, showing that temperature has a weak influence on ordering while electrolyte concentration and applied voltage can modify the pore arrangement (Ba \& $\mathrm{Li}, 2000)$. Thanks to SEM analysis $S(q)$ can be determined and used during the SANS fitting.

\subsection{Canceling of multiple scattering}

SANS is a powerful technique to characterize both the pore organization and the chemical composition by determining the different SLDs of the material through a fit of the experimental data. However, as mentioned in the Introduction, careful measurements are required prior to any fits of the SANS data to provide suitable conditions for data interpretation.

In particular, experimental conditions have to be chosen to cancel out MS, because it has a strong influence on the scattering intensity $I(q)$. A simple approach to detect MS is to measure the neutron transmission $\left(\operatorname{Tr}=N_{\mathrm{tr}} / N_{0}\right.$, with $N_{\text {tr }}$ the number of neutrons transmitted through the $\mathrm{AAO}$ and $N_{0}$ the number of neutrons of the incident beam) of the AAO (Cousin, 2015). $\operatorname{Tr}$ is defined as $\operatorname{Tr}=\exp \left(-n \sigma_{\text {tot }} L\right)$, with $n$ the number density $\left[=(\rho / M) N_{\mathrm{a}}, \rho\right.$ being the mass density, $M$ the molar mass and $N_{\mathrm{a}}$ the Avogadro number], $\sigma_{\text {tot }}=17.25$ barns the absorption cross section of alumina and $L$ the AAO length. Thus, the expected $\operatorname{Tr}$ for pure $\mathrm{Al}_{2} \mathrm{O}_{3}$ varies from 0.9998 to 0.9948 for the studied length range [see Fig. 2(a)].

Fig. 2(a) shows the Tr evolution of AAOs measured in air (red circles) as a function of AAO length. One can observe a substantial decrease in $\operatorname{Tr}$ with $L$ compared with the theoretical values. Since the adsorption cross section of alumina is low, such deviations come from MS. Only the very thin AAO membrane (below $6 \mu \mathrm{m}$ ) synthesized for this measurement has a high Tr in air, but such a thickness is in practice very difficult to handle. Another approach to avoid MS is to work with solvents having SLDs close to that of the AAO, i.e. close to the AAO matching point. Fig. 2(b) shows the Tr variation with the SLD of the solvent (i.e. the $\mathrm{D}_{2} \mathrm{O}$ volume fraction) for OA-18-11. A maximum in $\operatorname{Tr}$ of 0.917 is obtained for $73.2 \%$ $\mathrm{D}_{2} \mathrm{O}$, corresponding to an SLD of $4.53 \times 10^{-6} \AA^{-2}$. When we move away from this $\mathrm{D}_{2} \mathrm{O}$ proportion, $\mathrm{Tr}$ strongly decreases to reach 0.043 in pure $\mathrm{H}_{2} \mathrm{O}$ (SLD of $-0.56 \times 10^{-6} \AA^{-2}$ ). Few contrast conditions have $\operatorname{Tr}$ close to 0.9 , indicating that there is a small contrast range barely affected by MS. Therefore, in the following, we chose to work in $73.2 \% \mathrm{D}_{2} \mathrm{O}$ to minimize the MS effects whatever the AAO thickness, as shown in Fig. 2(a) (blue squares).
Now we propose to look at the scattering intensities $I(q)$ under several contrasts. Fig. $2(c)$ shows the $I(q)$ of OA-18-11 $\left(L_{\mathrm{p}}=171 \mu \mathrm{m}\right)$ immersed in different $\mathrm{H}_{2} \mathrm{O} / \mathrm{D}_{2} \mathrm{O}$ mixtures $(0$, i.e. in air, 73.2, 75.5 and $100 \% \mathrm{D}_{2} \mathrm{O}$ ). For $73.2 \% \mathrm{D}_{2} \mathrm{O}$, where $\mathrm{Tr}$ is maximum and thus MS absent, the SANS signal is characteristic of aligned cylinders parallel to the neutron beam with a typical $q^{-3}$ dependence at high $q$. At low $q$, a peak (located at the position $q^{*}$ ) is visible and originates from the pore-pore correlations [i.e. the signature of $S(q)]$. From the
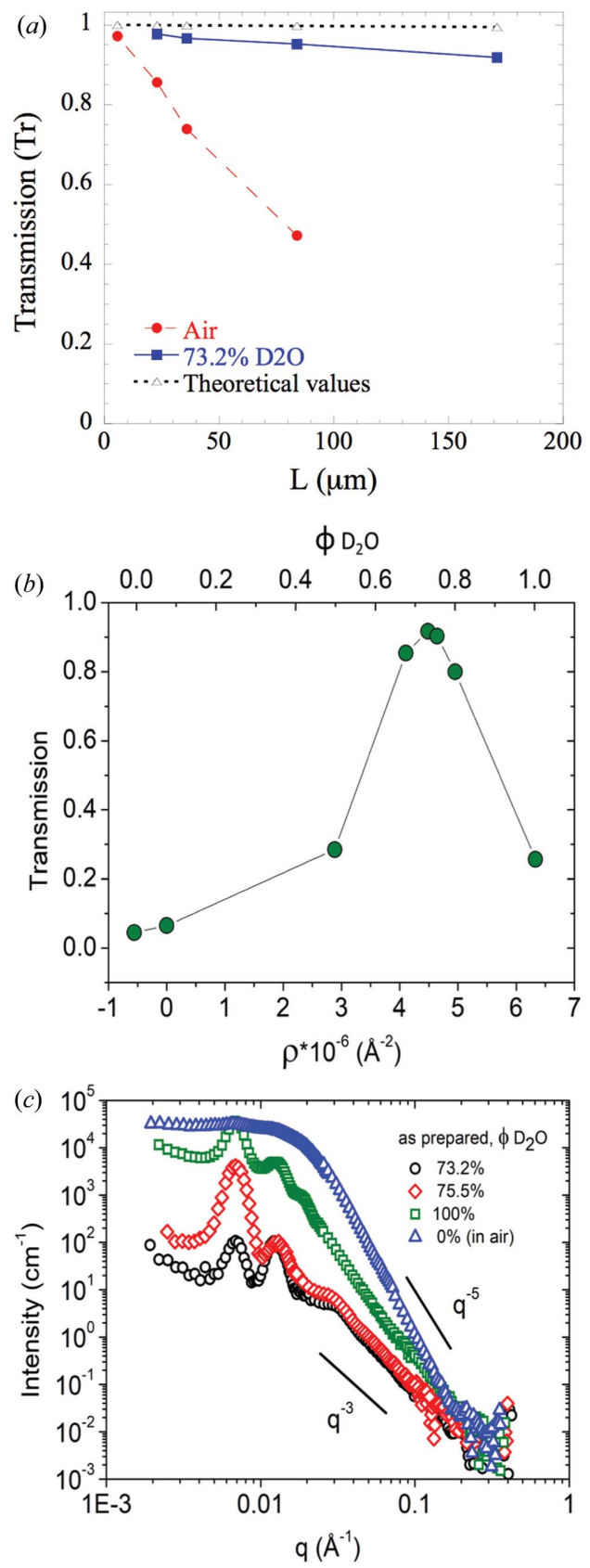

Figure 2

(a) Transmission evolution as a function of AAO length in air (red circles) and in $73.2 \% \mathrm{D}_{2} \mathrm{O}$ solvent (blue squares). The theoretical $\mathrm{Tr}$ values are plotted as black triangles. (b) Transmission variation with SLD (denoted as $\rho$ ). (c) Scattering intensities of AAO OA-18-11 for different $\mathrm{D}_{2} \mathrm{O}$ volume fractions: $0 \%$ (blue triangles), $73.2 \%$ (black circles), $75.5 \%$ (red diamonds), $100 \%$ (green squares). 
position $q^{*}$ the interpore distance $D_{\text {int }}$ can be calculated. In the intermediate $q$ range, different oscillations are observed coming from $S(q)$ and the form factor $\left\langle F(q)^{2}\right\rangle$ of the pores. For $75.5 \% \mathrm{D}_{2} \mathrm{O}$, the SANS scattering has the same general behavior at high $q$ and the same peak positions but shows an increase of the first peak amplitude, while the second one decreases. At $100 \% \mathrm{D}_{2} \mathrm{O}, I(q)$ in the intermediate $q$ range increases significantly compared with that of the $73.2 \% \mathrm{D}_{2} \mathrm{O}$ sample, with a $q^{-5}$ scaling and less pronounced peak amplitudes at low $q$. Note that all the peaks remain at the same position whatever the $\mathrm{H}_{2} \mathrm{O} / \mathrm{D}_{2} \mathrm{O}$ mixture. Since the theoretical $\mathrm{AAO}$ absorption cross section is low and $\mathrm{AAO}$ is well aligned, this increase is due to MS and is consistent with a low Tr. For $0 \% \mathrm{D}_{2} \mathrm{O}$ the $\mathrm{MS}$ is so pronounced that all peaks disappear and the intensity reaches values larger then $10^{4} \mathrm{~cm}^{-1}$. This additional $q$-dependent contribution caused by MS is due to the high anisotropic structure of the AAO. When MS occurs, the first scattering event occurs at an incident wavevector parallel to the pore axis. The beam is then scattered (mostly at the first correlation peak), leading to tilted incident wavevectors for the following scattering events. The Porod regime for tilted cylinders scales as $q^{-5}$, which is in good agreement with the $q$ dependency observed experimentally under MS conditions.

Finally, a low $\operatorname{Tr}$ and a deviation from the $q^{-3}$ behavior at high $q$ are the two criteria that can provide clear evidence of MS. As a consequence, to avoid MS, we describe our fitting strategy on SANS data measured in $73.2 \% \mathrm{D}_{2} \mathrm{O}$.

\subsection{Full SANS interpretation: fitting strategy}

In the following a detailed strategy will be presented to fully fit the SANS data in $73.2 \% \mathrm{D}_{2} \mathrm{O}$ (i.e. without the presence of MS) assuming that AAO can be described with a core/shell aligned-cylinder model. Such an approach has not been attempted so far owing to the complexity of obtaining a reliable SANS spectrum as described above.

3.3.1. Evidence of a contamination layer and estimation of its extent. As experimentally observed, the AAO membranes are not homogeneous in composition. Contamination from electrolyte anions creates compositional heterogeneities in the AAOs. The porous AAO cell is separated into two layers with different composition (Thompson \& Wood, 1981; Ono \& Masuko, 1992). The first layer is closer to the pore channel and consists of alumina which is contaminated with electrolyte roots such as oxalates, sulfates or phosphates depending on the acid used for the synthesis (Thompson \& Wood, 1981; Le Coz et al., 2010), and the amount and extent of root incorporation increases with the concentration of the electrolyte and is proportional to the applied voltage (MínguezBacho et al., 2015). The second layer consists of higher-purity alumina and extends to the skeleton of the porous structure.

This contamination (here oxalates, $\mathrm{C}_{2} \mathrm{O}_{4}{ }^{2-}$, since we used $\mathrm{OA}$ as electrolyte) can be shown by AAO dissolution in phosphoric acid $\left(5 \mathrm{wt} \%\right.$ at $30^{\circ} \mathrm{C}$ ) (Han et al., 2007). Fig. 3 presents the evolution of the pore diameter $D_{\mathrm{p}}$ determined by SEM (blue squares) of OA-18-11 as a function of the etching time in acid. Its evolution can reveal changes in the etching

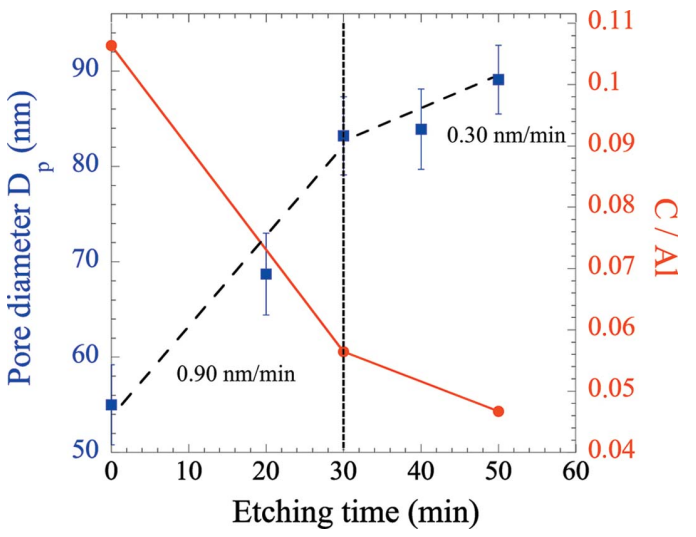

Figure 3

Evolution of pore diameter (blue squares) determined by SEM and C/Al ratio (red circles) determined by EDX with etching time in phosphoric acid $\left(5 \mathrm{wt} \%\right.$ at $\left.30^{\circ} \mathrm{C}\right)$ of a sample equivalent to OA-18-11.

dissolution rate which are related to the anion incorporation gradient along the pore wall. It is known that the anioncontaminated oxide has a higher solubility than the rest of the inner layer (Han et al., 2007; Lee \& Park, 2014). From the $D_{\mathrm{p}}$ evolution in Fig. 3, two regimes can be distinguished, and the slopes give the dissolution rates of the different porous alumina regions.

A decrease in the dissolution rate occurs after $30 \mathrm{~min}$ (passing from 0.9 to $0.3 \mathrm{~nm} \mathrm{~min}{ }^{-1}$ ), indicating the crossover between the contaminated layer (high dissolution rate due to the presence of contaminated anions) and the higher-purity alumina (lower dissolution rate). The extent of the anionincorporated layer can be estimated by the difference between the initial $D_{\mathrm{p}}(t=0)$ and that at the change of the slope $(t=$ $30 \mathrm{~min}$ ), giving a thickness of about $15 \mathrm{~nm}$ for the incorporated layer. This observation is confirmed by further EDX spectroscopy (red circles), showing a decrease of the C/Al ratio as the pore wall gets thinner, the carbon content being directly related to the amount of incorporated oxalate (see the elemental analysis in the supporting information). This experiment showing two distinct compositional regimes indicates that a core/shell cylinder model is suitable for the SANS fitting.

3.3.2. Description of the fitting model. The core/shell cylinder model is depicted in Fig. 4. The core corresponds to the center of the nanopores filled here by the solvent $(73.2 \%$ $\mathrm{D}_{2} \mathrm{O}$ ). The shell corresponds to the contaminated area and the bulk is the purer oxide.

In this model the form factor scattering amplitude for a perfectly aligned cylinder is given by

$$
F(q)=\left(\rho_{\text {solv }}-\rho_{\mathrm{s}}\right) V_{\mathrm{c}} \frac{2 J_{1}\left(q R_{\mathrm{p}}\right)}{q R_{\mathrm{p}}}+\left(\rho_{\mathrm{s}}-\rho_{\text {bulk }}\right) V_{\mathrm{s}} \frac{2 J_{1}\left[q\left(R_{\mathrm{p}}+t\right)\right]}{q\left(R_{\mathrm{p}}+t\right)}
$$

with $\rho_{\text {solv }}, \rho_{\mathrm{s}}$ and $\rho_{\text {bulk }}$ the SLDs of the solvent, the shell and the bulk, respectively. $V_{\mathrm{c}}$ is the volume of the core $\left(V_{\mathrm{c}}=\right.$ $\pi R_{\mathrm{p}}^{2} L_{\mathrm{p}}$, with $L_{\mathrm{p}}$ the length of the cylindrical object) and $V_{\mathrm{s}}$ the 
volume of the core/shell $\left[V_{\mathrm{s}}=\pi\left(R_{\mathrm{p}}+t\right)^{2} L_{\mathrm{p}}\right.$, with $t$ the shell thickness]. $J_{1}$ is the first-order Bessel function of the first kind.

The polydispersity in the radius and the shell thickness can also be taken into account with a Gaussian distribution given by the following equation:

$$
\left\langle F^{2}(q,\langle x\rangle)\right\rangle=\int F^{2}(q, x) f(x, \sigma) \mathrm{d} x
$$

with

$$
f(x, \sigma)=\frac{1}{(2 \pi)^{1 / 2} \sigma} \exp \left[-\frac{(x-\langle x\rangle)^{2}}{2 \sigma^{2}}\right] .
$$

$\langle x\rangle$ is the mean value of either the pore radius $R_{\mathrm{p}}$ or the shell thickness $t$, and $\sigma$ is the standard deviation (denoted as $\sigma_{\mathrm{p}}$ or $\sigma_{\mathrm{t}}$ for the radius or shell thickness, respectively). Finally the instrumental resolution was also accounted for through a resolution function $R(q,\langle q\rangle)$ (Lairez, 1999) as

$$
I(\langle q\rangle)=\int R(q,\langle q\rangle) I(q) \mathrm{d} q .
$$

In order to avoid any overlapping of the scattering objects, the shell thickness must be smaller than

$$
t<\left(D_{\text {int }}-D_{\mathrm{p}}\right) / 2 \text {. }
$$

For $\delta$, which quantifies the domain size $D$, we have to consider the coherence length of the neutrons in the transverse direction relative to the beam path, giving the maximum probing length of our sample, i.e. the maximum extent in which the neutrons can interfere (Grigoriev et al., 2010). Here, the value of $\delta$ will influence the SANS peak shape in the low- $q$ region [see Figs. 5(a) and 6]. The transverse coherent length of the neutron beam is given by

$$
L_{\mathrm{T}}=\lambda / \Delta \Theta,
$$

with $\lambda$ the neutron wavelength and $\Delta \Theta$ the divergence of the direct beam given by the experimental collimation (Lairez, 2010). Here, two cases have to be considered. If the domain size $D$ of the ordered domains as measured by SEM is smaller than $L_{\mathrm{T}}$ then the parameter $\delta$ will be fixed to that obtained from SEM. If the domain size $D$ is larger than $L_{\mathrm{T}}$ then $\delta$ is fixed to the minimal value set by the experimental collimation (see Table S2, which summarizes the different values depending on the instrumental configurations used during our

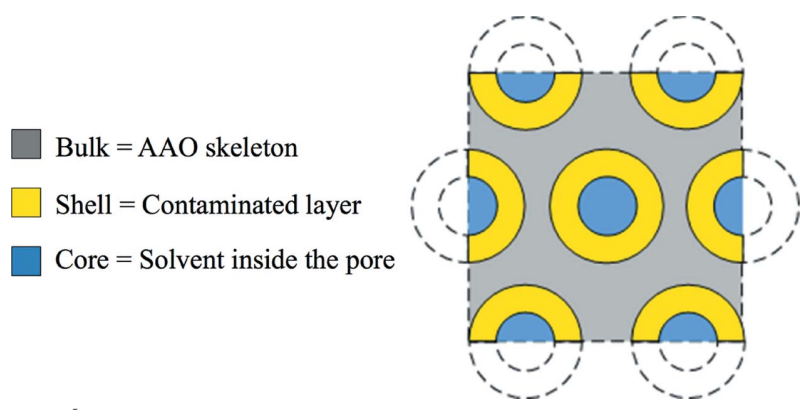

Figure 4

Schematic representation of the core/shell model: the nanopores, corresponding to the core in the fitting model, are filled with solvent (in blue) and the heterogeneity in composition is represented by a shell (in yellow). The remaining skeleton oxide in gray corresponds to the bulk in the model.
SANS runs). The values of $L_{\mathrm{T}}(>1000 \mathrm{~nm})$ are always larger than $D$, and thus the parameter $\delta$ will be fixed to the value determined by SEM analysis.

In the longitudinal direction similar considerations have to be taken into account. Since this length is critical for the SANS fitting, its determination is described in detail in Section 3.3.3.

Finally, the model is composed of many parameters summarized in Table 3. To reduce the number of fitting parameters we will fix all those extracted from the SEM analysis and will keep as variable $c_{\mathrm{L}}, \rho_{\text {shell }}, \rho_{\text {bulk }}, R_{\mathrm{p}}$ and $t$. Note that $R_{\mathrm{p}}$ remains a fitting parameter since the value extracted from SEM is generally larger than the actual SANS value
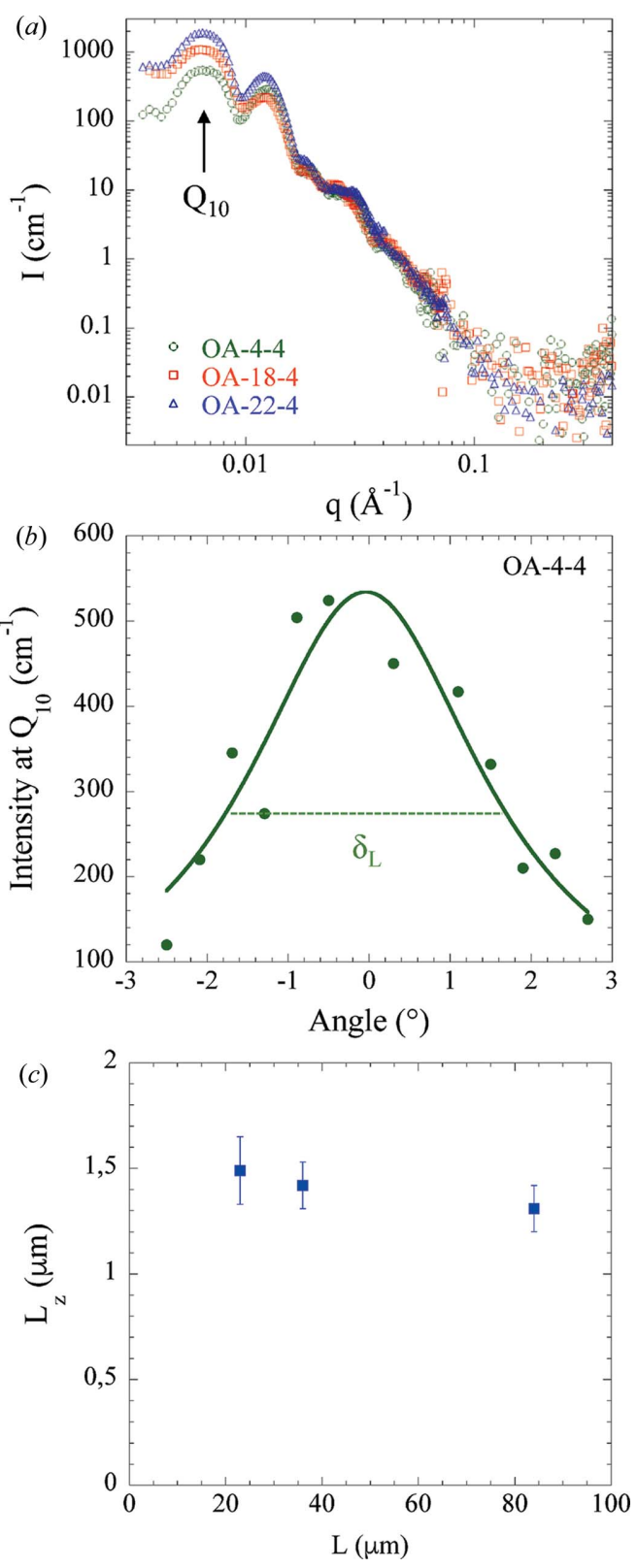

Figure 5

(a) SANS scattering intensities $I(q)$ of OA-4-4, OA-10-4 and OA-22-4, having different thicknesses ( $L=23,36$ and $84 \mu \mathrm{m}$, respectively). (b) Rocking curve of OA-4-4 fitted with a Lorentzian distribution (continuous line). (c) Longitudinal correlation length $L_{z}$ as a function of $L$. 
Table 3

Summary of all the parameters present in the model coming from the structure factor $S(q)$ or the form factor $\left\langle F(q)^{2}\right\rangle$.

Parameters that were fixed during the SANS analysis are marked with an asterisk (*).

\begin{tabular}{lll}
\hline Parameters & & $F(q)$ or $S(q)$ \\
\hline$* D_{\text {int }}$ & Interpore distance & $S(q)$ \\
$* \sigma_{a}$ & Relative interpore distance distribution & $S(q)$ \\
$* \delta / D$ & Peak width/domain size & $S(q)$ \\
$* m_{h k}$ & Peak multiplicity & $S(q)$ \\
$* c_{\mathrm{L}}$ & Correction factor for Porod invariant & $S(q)$ \\
$\phi_{\mathrm{s}}=n \pi\left(R_{p}+t\right)^{2}$ & Surface porosity & $F(q)$ \\
$* \rho_{\text {solvent }}$ & Solvent scattering length density & $F(q)$ \\
$\rho_{\text {shell }}$ & Shell scattering length density & $F(q)$ \\
$\rho_{\text {bulk }}$ & Bulk scattering length density & $F(q)$ \\
$R_{\mathrm{p}} / * \sigma_{\mathrm{p}}$ & Pore radius/pore standard deviation & $F(q)$ \\
$t * \sigma_{\mathrm{t}}$ & Shell thickness/shell standard deviation & $F(q)$ \\
$* L_{\mathrm{p}}$ & Cylinder length & $F(q)$ \\
\hline
\end{tabular}

because of the chemical etching during the AAO synthesis (Lee \& Park, 2014; Christoulaki et al., 2019). The data fitting was performed using the SasView software (SAS, http:// www.sasview.org/) with homemade programs.

3.3.3. Determination of the longitudinal correlation length $\boldsymbol{L}_{z}$. In principle, the fitting of the SANS data in absolute units

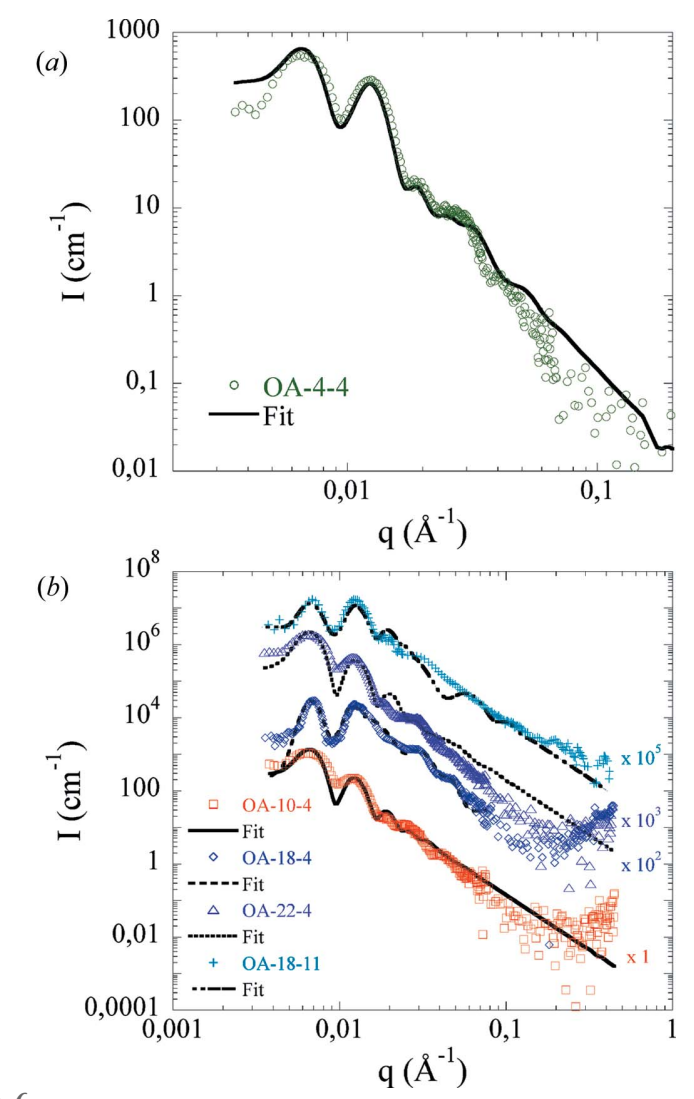

Figure 6

(a) SANS scattering intensity of OA-4-4 in $73.2 \% \mathrm{D}_{2} \mathrm{O}$. The continuous black line corresponds to the best fit. (b) SANS scattering intensity of OA-10-4 (red squares), OA-18-4 (blue diamonds), OA-22-4 (purple triangles) and OA-18-11 (light-blue crosses) in $73.2 \% \mathrm{D}_{2} \mathrm{O}$. The lines correspond to the best fits. The curves have been shifted for clarity by the factor given on the right. using the core/shell cylinder model described above can give a full description (structure and composition) of the AAOs. In this model the scattering intensity depends on the length of the AAO [see equation (5)]. However, a particular observation has to be made. Here we will focus on samples OA-4-4, OA-10-4 and OA-22-4 because they have been measured during the same SANS run, i.e. they have the same data resolution. Fig. 5(a) shows that the scattering intensities $I(q)$ of AAOs with different lengths $L(23,36$ and $84 \mu \mathrm{m}$, corresponding to OA-4-4, OA-10-4 and OA-22-4, respectively) superimpose at high $q$, suggesting that the probed length during the SANS experiments is the same whatever the real channel length $L$. Note that the larger differences in $I(q)$ at low $q$ might arise from differences in AAO composition and structure when the anodization temperature is increased to produce thicker AAOs. Grigoriev et al. (2007) proposed that the probed cylinder length to put in the SANS fitting is the longitudinal correlation length $L_{z}$ of the lattice, i.e. the ordering length along the pore channel. $L_{z}$ is determined by measuring the variation in the scattered intensity of the $Q_{10}$ component parallel to the cylinder axis $Q_{z}$ as a function of the tilting angle of the pore axis with respect to the incident beam. $L_{z}$ is then defined as

$$
L_{z}=\frac{2 \pi}{\Delta Q_{z}}=\frac{2 \pi}{Q_{10} \sin \delta_{\mathrm{L}}},
$$

where $Q_{10}$ is the 10 correlation peak position and $\delta_{\mathrm{L}}$ the width at half-maximum of the rocking curve.

It must be emphasized that such a procedure is no longer valid when $\mathrm{MS}$ occurs, justifying the use of the $73.2 \% \mathrm{D}_{2} \mathrm{O}$ solvent that cancels out MS (see above). Fig. 5(b) shows the rocking curve of OA-4-4 fitted with a Lorentzian distribution, and, using equation (11), a value of $L_{z}=1.49 \mu \mathrm{m}$ has been found. $L_{z}$ corresponds to the ordering distance, or grain size, in the longitudinal direction, i.e. along the pore channel. This value is of the same order of magnitude as the transverse size $D(0.66 \mu \mathrm{m}$ for OA-4-4) determined previously. Fig. 5(c) shows the different $L_{z}$ obtained for OA-4-4, OA-10-4 and OA-22-4. $L_{z}$ appears to be independent of the real length $L$ of the AAOs, an observation consistent with the superimposition of $I(q)$ in Fig. 5(a). Similar analyses have been performed for OA-18-4 and OA-18-11, and $L_{z}$ values of 1.74 and $1.33 \mu \mathrm{m}$, respectively, have been found. These results show that $L_{z}$ is a critical parameter to fully interpret the SANS data and, in the following fitting, we will use the values of $L_{z}$ as the length in equation (5).

3.3.4. Application of the fitting model to AAOs. The described strategy has been used to fit AAOs prepared in oxalic acid under different conditions as presented in Table 1. In particular, this approach can be used to quantify the effect of anodization conditions (time and temperature) on the AAO structure and composition by following both the form factor $\left\langle F^{2}(q)\right\rangle$ and the structure factor $S(q)$.

Fig. 6(a) shows the scattering intensity of OA-4-4 and the corresponding fit obtained by applying our strategy. The parameters derived from the fit are presented in Table 4. The asterisks $(*)$ indicate parameters that are fixed during the 
Table 4

Parameters obtained by the fitting of SANS data in Fig. 6 .

Parameters that were fixed during the SANS fitting are marked with an asterisk (*).

\begin{tabular}{lllllll}
\hline Name & \multicolumn{7}{c}{ OA-4-4 OA-10-4 OA-18-4 OA-22-4 OA-18-11 OA-18-11-E } \\
\hline$* D_{\text {int }}(\mathrm{nm}) \dagger$ & 100.0 & 102.6 & 106.4 & 103.4 & 100.0 & 100.0 \\
$* \sigma_{a}$ & 0.076 & 0.072 & 0.063 & 0.059 & 0.06 & 0.06 \\
$* \delta\left(\times 10^{-4} \AA^{-1}\right)$ & 9.5 & 8.4 & 8.2 & 8.5 & 9.5 & 9.5 \\
$* m_{h k}$ & 3 & 3 & 3 & 3 & 3 & 3 \\
$c_{\mathrm{L}}$ & 10 & 10 & 10 & 10 & 10 & 10 \\
$* \phi_{\mathrm{s}}$ & 0.73 & 0.59 & 0.24 & 0.54 & 0.56 & 0.66 \\
$* \rho_{\text {solv }}\left(10^{-6} \AA^{-2}\right) \ddagger 4.52$ & 4.52 & 4.52 & 4.52 & 4.52 & 4.52 \\
$\rho_{\text {shell }}\left(10^{-6} \AA^{-2}\right) \ddagger$ & 4.58 & 4.54 & 4.71 & 4.55 & 4.58 & - \\
$\rho_{\text {bulk }}\left(10^{-6} \AA^{-2}\right) \ddagger$ & 4.46 & 4.40 & 4.65 & 4.39 & 4.50 & 4.44 \\
$R_{\mathrm{p}}(\mathrm{nm})$ & 15.6 & 16.6 & 17.4 & 22.7 & 26.7 & 44.5 \\
$* \sigma_{\mathrm{p}}$ & 0.16 & 0.08 & 0.09 & 0.05 & 0.14 & 0.04 \\
$t(\mathrm{~nm})$ & 30.0 & 27.0 & 10.0 & 18.6 & 15.0 & 0 \\
$* \sigma_{\mathrm{t}}$ & 0.10 & 0.10 & 0.10 & 0.10 & 0.10 & - \\
$* L_{z}(\mu \mathrm{m})$ & 1.49 & 1.42 & 1.74 & 1.40 & 1.33 & 1.33 \\
\hline
\end{tabular}

$\dagger$ For OA-4-4 and OA-18-11, $D_{\text {int }}$ was a fitting parameter. $\$$ The errors of the fit are below $0.01 \%$. The error of the solvent SLD is around $0.2 \%$, corresponding to an SLD variation of $\pm 0.01 \times 10^{-6} \AA^{-2}$. Thus all the SLD values are given with two digits.

SANS fitting. The relative thickness distribution $\sigma_{\mathrm{t}}$ has also been fixed to an arbitrary value of 0.1 (corresponding to a reasonable relative polydispersity of $10 \%$ ) because of the low sensitivity of the fit to this parameter.

It can be seen that the core/shell model nicely reproduces the experimental data, validating our fitting strategy. Moreover, the intensity cannot be modeled without a shell, confirming the existence of a contaminated layer. For OA-4-4, we find an $R_{\mathrm{p}}$ of $31.5 \mathrm{~nm}$ (close to the SEM value, around $10 \%$ lower) and a shell thickness of $30.4 \mathrm{~nm}$ with an SLD $(4.58 \times$ $\left.10^{-6} \AA^{-2}\right)$ larger than the bulk SLD $\left(4.47 \times 10^{-6} \AA^{-2}\right)$. Fig. 6(b) shows $I(q)$ of the other AAOs with the best corresponding fits. The curves have been shifted for clarity. For all AAOs the radius found by SANS is close to that determined by SEM. A shell is always necessary and its extent ranges from 10.0 to $30.0 \mathrm{~nm}$, consistent with previous studies (Ono \& Masuko, 1992; Han et al., 2013). Note that for OA-18-11 the shell thickness $(15 \mathrm{~nm})$ is consistent with the value estimated

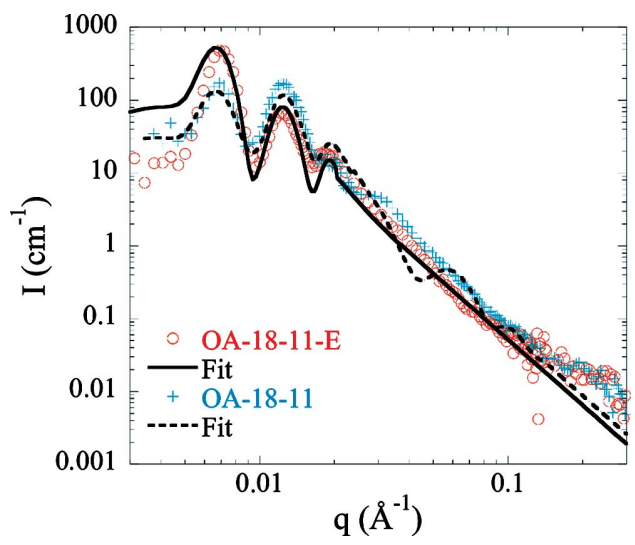

Figure 7

SANS scattering intensity of OA-18-11-E in $73.2 \% \mathrm{D}_{2} \mathrm{O}$ (orange circles). The continuous black line corresponds to the best fit. OA-18-11 SANS $I(q)$ with the fit is also represented for comparison. by the dissolution experiment (Fig. 3). This extent, as well as the shell and bulk SLDs, does not seem to be correlated to the current density, and the different values obtained for the bulk SLD are in the range of $4.4-4.65 \times 10^{-6} \AA^{-2}$, in good agreement with recent neutron reflectivity measurements (Christoulaki et al., 2019).

Then we can use the EDX data to determine the average density of the material for OA-18-11. From the SANS fit, the shell and bulk volume fraction can be determined and an average SLD of $4.55 \times 10^{-6} \AA^{-2}$ is calculated. Assuming that the material has an average chemical formula of $\mathrm{Al}_{2} \mathrm{O}_{3}\left(\mathrm{C}_{2} \mathrm{O}_{4}\right)_{x}$ (with $x=\mathrm{C} / \mathrm{Al}=0.106$; see Fig. 3), we calculate an average density of $2.976 \mathrm{~g} \mathrm{~cm}^{-3}$, consistent with a previous density determination (Abad et al., 2016).

All of the AAO samples show small SLD differences between the shell and the bulk, which actually have an important effect on the scattering intensity. The SLD depends on the composition of the material as

$$
\mathrm{SLD}=\frac{\rho N_{\mathrm{a}}}{M} \sum_{i} b_{i},
$$

with $\rho$ the density, $N_{\mathrm{a}}$ the Avogadro number, $M$ the molecular weight and $b_{i}$ the scattering length of atom $i$. A difference in SLD can be explained by a difference in composition or by a difference in the density of the materials. Here, the larger shell SLD can be related to the incorporation of $\mathrm{C}$ and $\mathrm{O}$ elements (through oxalates) having large $b_{i}$, thus increasing the shell SLD (Engel et al., 2009). Note that the incorporation of $\mathrm{OH}$ would have decreased the SLD since $\mathrm{H}$ has a negative $b_{i}$.

To further confirm the existence of the layer and validate its efficient determination through SANS fitting, an AAO equivalent to OA-18-11 was etched for $50 \mathrm{~min}$ in phosphoric acid $\left(5 \mathrm{wt} \%\right.$ at $\left.30^{\circ} \mathrm{C}\right)$. This chemical etching enlarges the pore diameter $\left(D_{\mathrm{p}}=89.1 \mathrm{~nm}, \sigma_{\mathrm{p}}=0.04\right.$, as observed in a SEM image; see Fig. S3) and dissolves the contaminated layer (as described previously in Fig. 3). The corresponding SANS of this etched sample (OA-18-11-E) is shown in Fig. 7 with the best fit. The as-prepared AAO (OA-18-11) is also plotted with the fit for comparison. While OA-18-11 needed a shell, the etched sample can be fitted without (see Table 4). In that case the only fitting parameter is the bulk SLD: we find a value of $4.44 \times 10^{-6} \AA^{-2}$, close to the bulk value before etching (i.e. $4.50 \times 10^{-6} \AA^{-2}$ ). Using $\mathrm{C} / \mathrm{Al}=0.0467$ (see Fig. 3 at $50 \mathrm{~min}$ ) we find a bulk density of $3 \mathrm{~g} \mathrm{~cm}^{-3}$, consistent with a higherpurity alumina region. Finally, from the SANS analysis, we can extract quantitative information about the composition of AAOs.

\section{Conclusions}

In this paper small-angle neutron scattering measurements coupled with scanning electron microscopy have been performed to gain understanding of the structure and composition of nanoporous alumina membranes. A strategy has been defined to measure AAOs in optimal conditions to avoid multiple scattering effects, and a core/shell cylinder 
fitting model was found to be appropriate to describe these systems. The existence of a layer contaminated by electrolyte anions (here oxalates) has been confirmed and quantified. We also found that the length in the fitting model is a critical parameter and corresponds to the longitudinal correlation length. Its determination is thus required to fit SANS data in absolute units. Finally, this original approach, based on a detailed and complete description of the SANS data, can be applied to AAOs prepared in different electrolytes (sulfuric or phosphoric acids) and extended to a variety of nanoporous media and will allow the further investigation of condensed matter under confinement.

\section{Acknowledgements}

The authors thank David Montero for his help during the SEM characterization and the Laboratoire Léon Brillouin (LLB) and Institut Laue-Langevin (ILL) for beamtime allocation.

\section{Funding information}

FEGSEM and EDX measurements were facilitated by the Institut des Matériaux de Paris Centre (IMPC FR2482) and funded by UPMC, CNRS and the C'Nano projects of the Région Ile-de-France.

\section{References}

Abad, B., Maiz, J. \& Martin-Gonzalez, M. (2016). J. Phys. Chem. C, 120, 5361-5370.

Anovitz, L. M. \& Cole, D. R. (2015). Rev. Mineral. Geochem. 80, 61164.

Ba, L. \& Li, W. S. (2000). J. Phys. D Appl. Phys. 33, 2527-2531.

Brûlet, A., Lairez, D., Lapp, A. \& Cotton, J.-P. (2007). J. Appl. Cryst. 40, 165-177.

Chenneviere, A., Bakangura, E., Christoulaki, A., Dubois, E., Grillo, I. \& Jouault, N. (2018). Conformation of Grafted Polymer Chains within Cylindrical Nanopores. Institut Laue-Langevin (ILL). https://doi.org/10.5291/ill-data.9-12-526.

Christoulaki, A., Chennevière, A., Dubois, E. \& Jouault, N. (2019). Nanoscale, 11, 2148-2152.

Cousin, F. (2015). EPJ Web Confer. 104, 01004.

Dewhurst, C. D. (2018). GRASP, https://www.ill.eu/en/users/supportlabs-infrastructure/software-scientific-tools/grasp/.

Dore, J. C., Benfield, R. E., Grandjean, D., Schmid, G., Krïll, M. \& Le Bolloc'h, D. (2002). Stud. Surf. Sci. Catal. 144, 163-170.

Engel, M., Stühn, B., Schneider, J. J., Cornelius, T. \& Naumann, M. (2009). Appl. Phys. A, 97, 99-108.

Förster, S., Timmann, A., Konrad, M., Schellbach, C., Meyer, A., Funari, S. S., Mulvaney, P. \& Knott, R. (2005). J. Phys. Chem. B, 109, 1347-1360.

Gommes, C. J., Prieto, G. \& de Jongh, P. E. (2016). J. Phys. Chem. C, 120, 1488-1506.

Goyal, P., King, J. S. \& Summerfield, G. (1983). Polymer, 24, 131-134.

Grigor'ev, S. V., Grigor'eva, N. A., Syromyatnikov, A. V., Napol'skii, K. S., Eliseev, A. A., Lukashin, A. V., Tret'yakov, Y. D. \& Eckerlebe, H. (2007). JETP Lett. 85, 449-453.

Grigoriev, S. V., Syromyatnikov, A. V., Chumakov, A. P., Grigoryeva, N. A., Napolskii, K. S., Roslyakov, I. V., Eliseev, A. A., Petukhov, A. V. \& Eckerlebe, H. (2010). Phys. Rev. B, 81, 125405.
Grillo, I. (2008). Soft Matter Characterization, edited by R. Borsali \& R. Pecora, pp. 723-782. Dordrecht: Springer.

Han, C. Y., Willing, G. A., Xiao, Z. \& Wang, H. H. (2007). Langmuir, 23, 1564-1568.

Han, H., Park, S.-J., Jang, J. S., Ryu, H., Kim, K. J., Baik, S. \& Lee, W. (2013). Appl. Mater. Interfaces, 5, 3441-3448.

Huber, P. (2015). J. Phys. Condens. Matter, 27, 103102.

Jouault, N., Chenneviére, A., Christoulaki, A., Dubois, E. \& Porcar, L. (2016). Polyelectrolytes Chain Conformation Under Confinement: Electrostatic Effects Studied By Zero Average Contrast Method. Institut Laue-Langevin (ILL). https://doi.org/10.5291/illdata.9-11-1764.

Krutyeva, M., Wischnewski, A., Monkenbusch, M., Willner, L., Maiz, J., Mijangos, C., Arbe, A., Colmenero, J., Radulescu, A., Holderer, O., Ohl, M. \& Richter, D. (2013). Phys. Rev. Lett. 110, 108303.

Lagrené, K. \& Zanotti, J.-M. (2008). MRS Proc. 1074, 1074-I13-02.

Lairez, D. (1999). J. Phys. IV, 9, Pr1-67-Pr1-81.

Lairez, D. (2010). Éc. Thémat. Soc. Fr. Neutron. 11, 43-69.

Le Coz, F., Arurault, L. \& Datas, L. (2010). Mater. Charact. 61, $283-$ 288.

Lee, W. \& Park, S. (2014). Chem. Rev. 114, 7487-7556.

Lefort, R., Duvail, J.-L., Corre, T., Zhao, Y. \& Morineau, D. (2011). Eur. Phys. J. E, 34, 71.

Marchal, D., Bourdillon, C. \& Demé, B. (2001). Langmuir, 17, 83138320.

Marchal, D. \& Demé, B. (2003). J. Appl. Cryst. 36, 713-717.

Masuda, H. \& Fukuda, K. (1995). Science, 268, 1466-1468.

Mata-Zamora, M. \& Saniger, J. (2005). Rev. Mex. Fís. 51, 502-509.

Md Jani, A. M., Losic, D. \& Voelcker, N. H. (2013). Prog. Mater. Sci. 58, 636-704.

Mínguez-Bacho, I., Rodríguez-López, S., Climent, A., Fichou, D., Vázquez, M. \& Hernández-Vélez, M. (2015). J. Phys. Chem. C, 119, 27392-27400.

Mozalev, A., Magaino, S. \& Imai, H. (2001). Electrochim. Acta, 46, 2825-2834.

Napolskii, K. S., Roslyakov, I. V., Eliseev, A. A., Petukhov, A. V., Byelov, D. V., Grigoryeva, N. A., Bouwman, W. G., Lukashin, A. V., Kvashnina, K. O., Chumakov, A. P. \& Grigoriev, S. V. (2010). J. Appl. Cryst. 43, 531-538.

Napolskii, K. S., Roslyakov, I. V., Romanchuk, A. Y., Kapitanova, O. O., Mankevich, A. S., Lebedev, V. A. \& Eliseev, A. A. (2012). J. Mater. Chem. 22, 11922-11926.

Ono, S. \& Masuko, N. (1992). Corros. Sci. 33, 503-507.

Pedersen, J. S. (1994). J. Appl. Cryst. 27, 595-608.

Richard, D., Ferrand, M. \& Kearley, G. (1996). J. Neutron Res. 4, $33-$ 39.

Rosenfeld, Y. (1990). Phys. Rev. A, 42, 5978-5989.

Roslyakov, I. V., Eliseev, A. A., Yakovenko, E. V., Zabelin, A. V. \& Napolskii, K. S. (2013). J. Appl. Cryst. 46, 1705-1710.

Roslyakov, I. V., Koshkodaev, D. S., Eliseev, A. A., Hermida-Merino, D., Petukhov, A. V. \& Napolskii, K. S. (2016). J. Phys. Chem. C, 120, 19698-19704.

Shin, K., Obukhov, S., Chen, J. T., Huh, J., Hwang, Y., Mok, S., Dobriyal, P., Thiyagarajan, P. \& Russell, T. P. (2007). Nat. Mater. 6, 961-965.

Sousa, C. T., Leitao, D. C., Proenca, M. P., Ventura, J., Pereira, A. M. \& Araujo, J. P. (2014). Appl. Phys. Rev. 1, 031102.

Sundblom, A., Oliveira, C. L. P., Palmqvist, A. E. C. \& Pedersen, J. S. (2009). J. Phys. Chem. C, 113, 7706-7713.

Thompson, G. \& Wood, G. (1981). Nature, 290, 230-232.

Turkevych, I., Ryukhtin, V., Garamus, V., Kato, S., Takamasu, T., Kido, G. \& Kondo, M. (2012). Nanotechnology, 23, 325606.

Waheed, A., Mehmood, M., Benfield, R., Ahmad, J., Amenitsch, H., Aslam, M., Rauf, A. \& Hassan, M. (2011). Mater. Chem. Phys. 131, 362-369.

Xiao, K., Wen, L. \& Jiang, L. (2016). Small, 12, 2810-2831. 